\title{
Amistad y relaciones sociales en el Mundo Antiguo. Una aproximación al fenómeno en el mundo griego y romano
}

\author{
Friendship and social relationship in the Ancient World. \\ A survey in the Greek and Roman World
}

\author{
BEgoña ENJUTO SÁNCHEZ*
}

\begin{abstract}
RESUMEN
El trabajo es una introducción a la mesa redonda desarrollada en el VII Encuentro de Jóvenes Investigadores, "Amistad y relaciones sociales en el Mundo Antiguo", donde el tema de debate fue la amistad como forma de relación social en el mundo griego y romano.
\end{abstract}

PALABRAS CLAVE:

Amistad, philía, amicitia, relaciones sociales, Grecia, Roma.

\begin{abstract}
This paper is the introduction to the panel «Friendship and social relationship in the Ancient World", celebrated in the VII Meeting of Postgraduate Students. The topic was friendship as social relationship in the Greek and Roman world.
\end{abstract}

KEYWORDS:

Friendship, philía, amicitia, social relations, Greece, Rome.

En el seno de cualquier sociedad distinguimos una forma de vinculación entre individuos no pertenecientes al mismo grupo de parentesco que identificamos con el término amistad. Este tipo de relación ha sido objeto de un creciente interés entre los estudiosos de Historia Antigua en los últimos decenios del siglo XX, como evidencia el importante incremento de publicaciones sobre tal argumento'. La mayoría de estos trabajos continuaban abordando esta temática como si se tratase de una cuestión de tipo filosófico, filológico o literario, dejando en un segundo

\footnotetext{
* Departamento de Prehistoria, H. Antigua y Arqueología, Universidad de Salamanca (C/ Cerrada de Serranos, s/n. 37008 Salamanca). E-mail: begens@ usal.es.

Artículo basado en la comunicación leída el 20 de Mayo de 2008, en la VII edición del Encuentro de Jóvenes Investigadores de Historia Antigua de la Universidad Complutense de Madrid.

1 Pizzolato (1993), Furst (1996), Fitzgerald (1997), Konstan (1997).
} 
plano la interesante cuestión de las relaciones interpersonales que a diario desarrollaba la práctica de la amistad².

Al hilo de estas últimas tendencias historiográficas, dentro del VII Encuentro de Jóvenes Investigadores, nos propusimos desarrollar una mesa redonda en la que se plantease debatir los aspectos sociales que la relación de amistad presentaba en el Mundo Antiguo. Este amplio espectro cronológico y geográfico pronto se vio delimitado. Casi todas las propuestas recibidas se circunscribían al ámbito de la historia de Roma, siendo minoritarias aquellas que presentaban su escenario de análisis en el mundo griego.

Si buscamos en el diccionario de la Real Academia de la Lengua Española el significado de la palabra amistad, la primera acepción que presenta el vocablo es: «Afecto personal, puro y desinteresado, ordinariamente recíproco, que nace y se fortalece con el trato». De dicha definición es importante destacar el carácter ético y social que entraña y desarrolla dicho afecto. Son estas características las que nos permiten no quedarnos en el estricto sentimiento e ir más allá, posibilitando estudios de carácter histórico, filosófico o social. Son esas implicaciones, principalmente las de carácter social —aunque aparezcan veladas bajo otro tipo de elementos-, sobre las que queremos reclamar la atención.

Esta inicial declaración de intenciones y objetivos no es óbice para que, en nuestras investigaciones, descubramos que bajo el concepto de amistad existen importantes conexiones con ciertas consideraciones de carácter ético, político e incluso ideológico que han de exponerse y aclararse en sus justos términos. Su utilización y aplicación en contextos como la diplomacia, la política o la ética nos revelan, de manera significativa, la forma en que una cultura - la griega o la romana-, concibió sus relaciones, tanto a nivel de comunidad, con otros pueblos, como de manera individual, y a título privado, entre sus integrantes. En este último caso, el estudio de las relaciones personales que se establecen en el seno de una sociedad posee un conjunto de interesantes factores, tales como la edad, el género, el estatus o la clase social a la que los individuos pertenecen, los cuales no podremos desatender en nuestros trabajos ya que determinaron el desarrollo de estos vínculos.

En otro orden de cosas, debemos señalar que a la hora de abordar estas investigaciones el historiador debe dejar a un lado la concepción que sobre este tipo de relación intergrupal posee, que viene a coincidir, grosso modo, con aquella que hemos visto aparecer en el DRAE. De otro modo, el estudioso no captará la variedad de matices, conexiones y vinculaciones que vocablos como philía o amicitia poseen en el panorama social greco-romano. Además cometerá un importante error metodológico, puesto que nuestro concepto de amistad es una compleja reelaboración de ideas, fruto de una serie de circunstancias históricas posteriores al mundo clásico greco-romano. Concretamente esta primera 'reconstrucción' hunde sus raíces en el Renacimiento, cuando se retomaron y adecuaron a la época los

2 Reali (1998), Peachin (2001). 
principios éticos y filosóficos aristotélicos sobre este fenómeno ${ }^{3}$. Pero no fue hasta los siglos XVIII y XIX cuando la llustración, el liberalismo... en definitiva, toda una serie de avatares históricos que sacudieron a Europa en ese periodo, contribuyeron a la construcción del actual concepto de amistad ${ }^{4}$.

La variedad de matices que sobre el concepto de amistad encontramos en el mundo griego y romano no nos inhibe de intentar presentar una noción básica que, reuniendo unos mínimos requisitos, se encuentre presente en cada uno de aquellos escenarios geográficos y temporales que vamos a estudiar.

Podríamos afirmar que, dentro de las relaciones sociales de carácter personal que se pueden desarrollar en cualquier sociedad, existe un tipo de vinculación entre individuos no pertenecientes al mismo grupo de parentesco, donde llama la atención la reciprocidad que se establece entre ellos. En las fuentes documentales donde se constatan tales relaciones, ese tipo de vinculación aparece designada a través de una serie de términos tales como philía, xenía, hetairía, en el caso del mundo griego, o amicitia, amicus en los documentos latinos, vocablos cuya traducción en las lenguas modernas se ha hecho corresponder con expresiones relacionadas con el campo semántico de la amistad 5 . Dentro de esta definición que acabamos de proponer hemos optado por no incluir dos elementos que en nuestra concepción actual soportan una parte importante de la peculiaridad de este tipo de relación: la afectividad, en definitiva, el sentimiento que incita a que se produzca tal vinculación de un modo desinteresado, y la libertad de elección para entablar amistad con otro individuo.

Si vaciamos el concepto de su parte sentimental o la reducimos a una mínima expresión, con nuestra acción anulamos en gran medida la actuación desinteresada. Los propósitos, las intenciones, en definitiva, las pretensiones que se establecen en la base de las relaciones de amistad se encuentran incentivadas, desde esta nueva perspectiva, por inquietudes políticas, económicas, ideológicas o de cualquier otra índole. En otras palabras, en última instancia estos vínculos buscan la consecución de una serie de beneficios, servicios, actuaciones comunes, etc., por parte de los individuos que entablan una amistad.

Bajo estos parámetros, los lazos de amistad comienzan a tomar una serie de matices que les asemejan a otro tipo de fenómenos sociales, como son el patrocinio y el clientelismo; formas de relación a las que tendremos que aludir, y lo más importante, justificar su diferenciación, en relación con aquellos procedimientos propios de la amistad 6 . De este modo, los resultados y balances de nuestras investigaciones no sólo contribuirán al esclarecimiento de las relaciones y los valores sociales en el seno del mundo griego y romano, sino que revertirán, además, en un mayor conocimiento de sus mentalidades.

3 Narducci (2007).

4 Silver (1990: 1484-90).

5 Hellegouarc'h (1972), Konstan (1997: 8-10).

6 Saller (1982:15-22), Evans (1997: 181), Konstan (1997: 137), Verboven (2002). 
Sobre la cuestión de la libertad hemos de señalar que éste es un elemento claramente influenciado por el pensamiento ilustrado, llevado hasta sus últimas consecuencias en los dos últimos siglos de nuestra historia. Por lo tanto, cabría preguntarnos hasta qué punto existe libertad para poder establecer este tipo de relaciones sociales en el mundo antiguo, concretamente en las sociedades griega y romana, sociedades fuertemente jerarquizadas en las que las relaciones entre sus diferentes estratos se encuentran tipificadas y reguladas por mecanismos de carácter económico y jurídico. En este sentido los testimonios de Cicerón o Séneca, ambos en el ámbito filosófico, demuestran las dificultades a este respecto ${ }^{7}$.

A tenor de estos planteamientos existen una serie de interrogantes sobre los que podemos debatir y podríamos formular bajo los siguientes parámetros: ¿cómo se definen e identifican las relaciones de amistad en el mundo griego y romano? ¿En qué escenarios las encontramos -epigrafía honorífica, funeraria, epístolas...-?, y en consecuencia, ¿cómo determinan las fuentes nuestros estudios? Además, ¿qué tipo de conductas sociales pautan este tipo de relación? Pero también podemos interrogarnos sobre sus protagonistas, y en este sentido, ¿únicamente podemos calificar de amistad un tipo de vinculación que exclusivamente se desarrolla entre individuos pertenecientes a un mismo grupo socio-jurídico? ¿El resto de vinculaciones con personajes de diferente estatus jurídico o social debe ser encasillado dentro de los fenómenos del clientelismo, patrocinio o la dependencia? En otras palabras, bajo el apelativo de amistad, ¿hacemos referencia a un tipo de relaciones que podemos denominar horizontales, de igualdad social entre sus protagonistas, o podemos vislumbrar una forma de expresar jerarquías en el seno de un mismo estatus social o jurídico? ¿Cómo deberíamos analizar esta vinculación social desde una perspectiva de género? ¿Podemos ver la amistad como una forma de cohesión social intra-grupal que tiene su mejor forma de expresión entre los grupos dirigentes?

Expuestos brevemente algunos de los puntos de reflexión que suscita el tema, retomemos las cuestiones relacionadas con las fuentes documentales que sustentan nuestros análisis. En este sentido hemos de señalar que el estudio de la amistad en el mundo grecorromano está basado casi exclusivamente en documentos literarios y epigráficos, a los que recientemente se ha añadido el análisis de los papiros.

Determinar los parámetros de la amistad en el mundo antiguo, desde esta perspectiva de la naturaleza de las fuentes, ha conducido a ciertos investigadores a desarrollar una serie de tareas de carácter filológico. Principalmente entre los estudiosos del mundo griego, pronto se advirtió que un concepto tan amplio y con tantas connotaciones como es el de amistad no puede circunscribirse a una única palabra específica ${ }^{8}$. De este modo, descubrimos que el sustantivo phílos, palabra griega que normalmente se traduce como 'amigo', también puede utilizarse, de una forma más amplia, para hacer referencia a ciertas relaciones de parentesco o a

7 Cic. Lael. 67-68, 70; Cic. Fin. 2.78; Sen. Brev. 7.7; Sen. Epist. 48.3.

8 Pizzolato (1993: 3). 
aquellas que se desarrollan entre ciudadanos ${ }^{9}$. Pero también existe otro tipo de vocablos, como xénos o hetaîros, que nos hablan de un tipo de relación cercana a la amistad que, sin embargo, presenta una serie de connotaciones de carácter económico, político o militar, que las individualizan, identifican y, por tanto, diferencian considerablemente de lo que podemos entender como philía.

En el caso del latín, los vocablos amicus y amicitia remiten a realidades que se aproximan a nuestros términos amigo y amistad, al hacer referencia a relaciones distintas a aquellas del parentesco, la etnicidad y la asociación utilitaria. La dificultad surge cuando tales vocablos aparecen en contextos políticos, jurídicos, ya que nos advierten de otro tipo de realidades sociales ${ }^{10}$. En esos casos nos encontramos ante una concepción de la amistad entendida como relación interesada, sobre todo desde el punto de vista político y social ${ }^{11}$. Dicho fenómeno podemos observarlo más allá del contexto geográfico de Roma y sus grupos dirigentes ${ }^{12}$, senadores y ecuestres, en época imperial, cobrando una especial relevancia en el ámbito provincial, en la promoción de las oligarquías locales o en la interesante cuestión de la integración social de los libertos ${ }^{13}$.

Por otra parte hay que señalar que el estudio de la amistad en las culturas griega y romana ha tenido un diferente tratamiento por parte de la historiografía. Podemos constatar que el interés de los investigadores del mundo griego se ha dirigido a analizar la philía desde parámetros literarios y filosóficos, siendo significativos los análisis de las obras de Aristóteles o las investigaciones en torno a este concepto contextualizado en las distintas escuelas filosóficas helenísti$\operatorname{cas}^{14}$. Los aspectos sociales han pasado a un segundo plano, y se han visto circunscritos al estudio de la xenía y la hetairía - tanto en contextos políticos como militares-, tendencia de la que han quedado excluidas las obras homéricas, donde se ha prestado una gran atención a las relaciones sociales desarrolladas por la philía ${ }^{15}$.

En el caso de la amicitia romana, los trabajos se han centrado principalmente en la etapa republicana y en dos ámbitos bien diferenciados, aunque incluidos dentro del contexto de la historia política. En primer lugar, se ha investigado este tipo de relaciones personales en los círculos de la nobilitas romana, fenómeno que se traduce en solidaridad política. Estos lazos interpersonales son la forma sobre la que se sustentaban unas sencillísimas formaciones políticas, precursoras de lo que entendemos hoy en día por facción o partido político ${ }^{16}$. En este sentido, el estudio de las obras de Cicerón ha sido paradigmático para tales trabajos, princi-

9 Whitlock (1989: 39).

10 Ej.: AE 1959, 0011 (1961, 0184). Cf. Caldelli (2001).

11 Rouland (1979).

12 Panciera (2001), Caldelli (2001).

13 Serrano Delgado (1987-1988: 351; 1988: 227-228).

14 Especial relevancia ha tenido la obra aristotélica, Ética a Nicómaco. Stern-Gilllet (1995), SavalliLestrade (1998).

15 Finley (1977), Donlan (1985: 233-244), Montes Miralles (2006).

16 Syme (1939:157). 
palmente el Laelius, donde se plantea la amistad desarrollada entre los boni, los sapientes. Nos encontramos ante un ensayo, un texto filosófico en el que el autor no solo proyecta cómo deberían ser las relaciones interpersonales, sino que hace gravitar el buen funcionamiento de la política internacional de Roma y la propia concordia ordinum de su sociedad sobre un correcto ejercicio de la amicitia, convirtiendo de este modo la amistad en un fenómeno totalizador de la experiencia humana ${ }^{17}$.

El segundo - aunque no por ello menos importante- objeto de análisis ha sido el estudio de la amistad en el ámbito de la política internacional romana. Las relaciones de paz, las alianzas que Roma estableció con sus socii, e incluso con sus hostes en calidad de aliados políticos, se presentan en ciertas ocasiones - sobre todo en época republicana- como una adaptación de determinados modos propios de la amicitia, utilizándose formas y vocabulario propio de este tipo de relación en un contexto público como la política exterior romana ${ }^{18}$.

El nuevo sistema sociopolítico inaugurado por Octaviano afectó ineludiblemente a ese mundo de las amistades políticas de época tardorrepublicana ${ }^{19}$. Con la incursión de la figura del princeps en el vértice de aquella jerarquizada sociedad, tanto las relaciones de poder que el Augusto entabla con los otros grupos dirigentes - senadores, ecuestres y decuriones-como las actividades y beneficia que aquellos buscan obtener de sus buenas relaciones con el emperador encuentran en el lenguaje de la amicitia un perfecto instrumento ideológico ${ }^{20}$. A tenor de esta nueva realidad, no debe extrañarnos que el consilium principis, el grupo de consejeros que rodea al emperador Adriano, pase a denominarse amicili, y que las fuentes jurídicas de la época ofrezcan datos relevantes sobre los officia amicitiae, los beneficia, las liberalitates amicorum, o la propia existencia de intermediarios profesionales, auténticos corredores que se ocupaban de proporcionar contactos y facilitar la consolidación de relaciones interpersonales ${ }^{22}$.

En consecuencia, entender la amistad en Roma como una relación recíproca e interesada entre los grupos dirigentes, principalmente en todo lo relacionado con la esfera política, es uno de los temas recurrentes que podemos encontrar en la historiografía moderna en relación a la historia social de los siglos I-II d.C ${ }^{23}$.

Dicha visión es fruto de las importantes investigaciones realizadas a las obras de autores como Séneca ${ }^{24}$, Marcial, Juvenal, Plinio el Joven, así como de los resultados obtenidos de los análisis realizados a los propios textos epigráficos altoimperiales. Estos últimos reflejan interesantísimas relaciones personales, tanto entre los diver-

17 Cic. Lael. 50. Schievenin (2000), Citroni (2000: 8).

18 Marchall (1968), De Martino (1973: 29-35), Cimma (1976: 21-32), Burton (2000).

19 Alföldy (1988: 138).

20 Plin. Paneg. 85,1-8. Winterling (2008).

21 Crook (1955: 21-30), Millar (1977).

22 D. $50.14,3$ (Ulpiano)

23 Brunt (1988: 352).

24 Epistulae ad Lucilium y, más concretamente De beneficiis de Séneca, son fundamentales para entender el concepto de amistad en el estoicismo. Saller (1989:49-62). 
sos colectivos jurídico-sociales -ingenuos, libertos, esclavos-como entre comunidades y ciertos individuos pertenecientes a los ordines romanos ${ }^{25}$.

En esta nueva dinámica del poder, las relaciones de amicitia entre los grupos dirigentes son un importante mecanismo de exhibición del prestigio y el poder. La importancia de estos vínculos se mantuvo a lo largo de las siguientes centurias, como bien ejemplifican las cartas comendaticias que han llegados hasta nosotros de autores como Plinio ${ }^{26}$, Símaco o Libanio.

La estrecha relación existente entre amistad, género epistolar y grupos nobiliarios parece no necesitar mayores explicaciones. La epístola, además de un ejercicio de habilidad retórica que por sí mismo denota el carácter culto, refinado, y en consecuencia elitista del autor y su destinatario, es, en sí misma, el vehículo perfecto para el mantenimiento de una red de contactos ${ }^{27}$. Así mismo, gracias a este procedimiento, se creaba un elemento de cohesión ideológica, de solidaridad entre aquellos individuos, reforzándose su sentimiento de pertenencia a un grupo privilegiado. Pero estas relaciones de amistad son también el medio a través del cual, a finales del siglo IV, se integran en las capas sociales altas los miembros más destacados de la burocracia y del ámbito militar, donde predominaban, en este último, los generales y comandantes de origen bárbaro ${ }^{28}$.

La amicitia en el mundo tardoantiguo ha sido analizada siempre desde la perspectiva del conflicto ideológico y religioso, desarrollado entre el paganismo y el cristianismo. La idea de amistad, como otros ideales éticos, religiosos y políticos romanos, se asentaba en la denominada mos maiorum, y por tanto, en la continuidad con los valores de la antigua Roma, que se contraponían a aquellos nuevos preceptos cristianos, como el amor fraternal o la caridad, los cuales se integraban en el nuevo concepto de amistad cristiana ${ }^{29}$.

Pero la tradicional amicitia, aquella que se desarrollaba entre los hombres de cultura, no parece conocer de credos ni sentimientos religiosos como nos ilustra la correspondencia entre Símaco, firme defensor del paganismo, y su contemporáneo Ambrosio, obispo de Milán ${ }^{30}$. A tenor de estos datos podríamos afirmar que la proliferación de este tipo de vinculación depende de elementos más sociales e ideológicos que propiamente religiosos. Los Padres de la Iglesia pertenecían a un grupo de intelectuales, muchos de ellos procedentes de importantes familias aristocráticas - caso del citado Ambrosio, o de Paulino de Nola-, quienes se habían educado, al igual que los más firmes defensores del paganismo, en un mismo sistema de valores transmitido a través de la paideia.

Este rápido recorrido sobre los tratamientos historiográficos y las aproximaciones más recurrentes que podemos encontrar en relación al tema de la amistad

\footnotetext{
25 Vid. supra n. 13.

26 Aubrion (1989), De Blois (2001).

27 Matthews (1974), Roda (1984).

28 Salzman (2006).

29 White (1992).

30 Symm. Epist. 2.30-37.
} 
en las culturas griega y romana ha pretendido ser un marco donde contextualizar las distintas aportaciones tratadas en la mesa redonda.

Nuestro foro de debate arranca presentando los banquetes griegos (sympósia) como un marco ideal para el estudio de las relaciones de amistad, al ser un lugar de encuentro y, por tanto, el escenario perfecto para el estudio de las relaciones interpersonales de la mano de Silvia Porres Caballero y su comunicación «Amistad y simposio en la poesía lírica». En este espacio doméstico, privado, se reúne una nutrida red de ciudadanos que, apelando a los lazos de philía, debaten cuestiones de índole política, social, filosófica... convirtiéndose en interesantes opúsculos de partidos políticos. Son los sympósia aquellos lugares donde se redefinen y consolidan los grupos de amigos, al mismo tiempo que, por exclusión a tales reuniones, se definen los adversarios políticos. Este tipo de encuentros desempeña, además, un importante objetivo social: lograr la cohesión interna de los grupos dirigentes. Al hilo de este discurso, la autora se interrogaba sobre la función que la poesía lírica dionisiaca cumplía en este tipo de eventos sociales. La recitación de cantos y poesías dedicadas a dicho dios, en definitiva, la ritualidad que aparece en los textos, se presenta como un interesante medio de consolidar vínculos entre ciudadanos, sancionados desde un ámbito religioso. Esta apelación al mundo divino, sin embargo, no tiene paralelo en Roma ya que el concepto de amicitia llevaba implícito la connotación religiosa de fides.

El mundo romano tuvo su escenario de debate centrado en dos periodos contrapuestos en su historia: el republicano — donde el tema principal fue el estudio de las interrelaciones entre política, amistad y relaciones clientelares-, y la Antigüedad Tardía, etapa en la que la amicitia fue analizada dentro de dos contextos: la conformación del poder episcopal y los conuiuia aristocráticos.

El papel desempeñado por Roma en el mundo helenístico del siglo II a.C. nos presenta una interesante situación en la que se combinan elementos de derecho político internacional y relaciones de amistad. Denis Álvarez Pérez Sostoa, a través de su trabajo «Escipión Africano Menor, Polibio y Demetrio I Sóter», se interroga sobre el papel jugado por la amicitia romana en la interpretación de las relaciones llevadas a cabo entre aristócratas romanos y aquellos individuos helénicos que, siendo confinados políticos en Roma, lograron, no obstante, entablar interesantes vínculos con las elites del momento. El caso de Escipión, Polibio y Demetrio planteado por el autor nos pone en contacto, además, con la realidad jurídica y social de los rehenes, los detenidos, quienes, curiosamente, aparecen en las fuentes literarias en calidad de invitados, como son calificados por Cicerón. De este modo descubrimos cómo los autores romanos encubren una realidad, la de los prisioneros de alto estatus social e intelectual. En este sentido, tal tratamiento trae a nuestra memoria el concepto de xenia del mundo griego, su adecuación al mundo helenístico y, en consecuencia, una aplicación sui generis en el contexto político romano del siglo II a.C.

La alianza entre Roma y otros pueblos siempre ha tenido en el ámbito de la amistad un importante punto de referencia a la hora de definir las relaciones exte- 
riores entre la Urbs y los otros. Sin embargo, no podemos olvidar las relaciones de clientelismo y sometimiento que la ciudad del Lacio ejerció como potencia vencedora a lo largo de su historia. En este sentido, un caso significativo en el seno de la sociedad romana republicana — donde se conjuga esta casuística, y en la que la amistad funcionó como vehículo de integración de los grupos de poder indígenasfue el desarrollo del bellum civile en Hispania. El trabajo presentado por Miguel Ángel Novillo López, «Amicitia y relaciones clientelares con pompeyanos y cesarianos durante el bellum hispaniense», subraya la existencia de dos modelos de integración del territorio hispano en la administración romana ideados por Pompeyo y César. En ambos casos, para comprender la integración de las elites locales dentro de los ámbitos de poder romanos debemos considerar el extraordinario papel de las relaciones de amistad, que necesitan ser distinguidas de otro importante fenómeno: el clientelismo.

La amistad también podría ser concebida como solidaridad mutua entre individuos, quienes, además, llegaron a organizarse en función de una serie de intereses de diversa índole -económica, social, religiosa-. Dentro de esta concepción del término, y en relación al mundo romano, la plebe se presenta como la gran protagonista de este tipo de fenómeno y, concretamente, la creación de los collegia su mejor forma de expresar este tipo de solidaridad. En este sentido, la contribución de Luis Manuel López Román, «Publio Clodio y la Lex de collegiis: una aproximación al fenómeno asociativo a finales de la República romana», nos acerca al siempre interesante aunque todavía poco estudiado tema de la historia de la plebe desde una innovadora perspectiva: las relaciones de solidaridad y la utilización política de los collegia. Estas asociaciones aparecen en las fuentes como las generadoras de importantes altercados y disturbios callejeros, si bien es cierto que en última instancia la responsabilidad de los hechos recaía en los aristócratas republicanos. Eran ellos quienes manipulaban las agrupaciones, hasta el extremo de dirigirlas al fanatismo, al furor que conduce a episodios de máxima tensión política, hecho que, en aquel momento, convierte la violencia en un instrumento de poder, en un elemento estructural del sistema.

El estudio de la amicitia en el ámbito de la Roma cristiana en nuestra mesa redonda viene de la mano de David Natal Villazala, quién, a través de su trabajo "Amicitia Episcoporum: Ambrosio de Milán y los obispados satélites del norte de Italia a finales del siglo IV», nos plantea el estudio de la amistad en los contextos episcopales, donde los lazos de amistad se manifiestan como un fenómeno a medio camino entre las relaciones privadas y la esfera pública, y, en consecuencia, siendo un interesante vehículo de ascensión social y eclesiástica. La creación de una red de partidarios en torno a la figura de Ambrosio en diferentes sedes episcopales del norte de Italia confirma la utilización de este tipo de relaciones para controlar un extenso territorio diocesano. Bajo esta perspectiva, la amicitia se presenta como una de las estrategias utilizadas por las nuevas élites eclesiásticas para suplir, desde el ámbito de la administración episcopal, los vacíos provocados por los procesos de fragmentación del poder imperial y aristocrático en el ámbito local y diocesano a finales del siglo IV. 
Finaliza nuestro foro de debate en un escenario aristocrático, de ámbito privado, aunque ahora en el contexto de la Roma tardoimperial, como son los conuiuia. Andrea Livini, con su comunicación «Septem conuiuium, nouem vero conuicium: Consideraciones sobre la importancia de los conuiuia nobiliarios tardo-antiguos», nos plantea el doble carácter social y político de estas reuniones, en cuyo desarrollo pautado sabemos del origen del nacimiento de un género literario creado ex profeso para tales banquetes. El auge de estas reuniones, además de manifestar el bienestar económico de un limitado grupo, se presenta para el investigador como una interesante forma de exhibir y ostentar el control político y social, principalmente entre las altas capas civiles y militares. $Y$ todo ello bajo los parámetros de la amicitia romana que, en este sentido, sirve además para cohesionar a los grupos dirigentes. Por otra parte, recordemos que estos conuiuia también se constituyen llegado el caso como el punto de reunión y encuentro de los grupos opositores al régimen imperial y, por tanto, se revelan como el escenario donde tienen lugar usurpaciones como las de Magnencio o Juliano en el siglo IV.

Me gustaría finalizar esta introducción expresando mi agradecimiento a los organizadores del VII Encuentro de Jóvenes Investigadores por haberme invitado a colaborar en la III Edición Nacional de estos encuentros, a través de la propuesta temática y posterior moderación de una de sus mesas redondas. Sin su apoyo y esfuerzo dicho encuentro no se hubiera podido llevar a cabo, ni hubiera sido posible la publicación de estas actas. En este sentido, sé de la inquebrantable voluntad y el gran empeño de Ana Rodríguez Mayorgas y Fernando Echeverría Rey por conseguir la publicación de nuestras contribuciones, propósito que se ha visto materializado con la inestimable ayuda de Javier Andreu. A ellos les agradezco profundamente todo sus esfuerzos, dedicación y trabajo. También querría aprovechar esta ocasión para manifestar mi más sincera gratitud al consejo de redacción de la revista Espacio, Tiempo y Forma por acceder a la publicación de los trabajos en su prestigiosa publicación periódica. Con tal decisión no sólo apoyan este tipo de iniciativas sino que, con su respaldo, nos brindan una importante oportunidad para presentar y difundir nuestros trabajos a nivel nacional e internacional. Su determinación evidencia su apuesta y confianza en nuestras investigaciones, en las nuevas generaciones.

\section{BIBLIOGRAFÍA}

ALFÖLDY, G. (1988), Historia Social de Roma, Madrid.

AUBRION, E. (1989), «La correspondance de Pline le Jeune: problèmes et orientations actualles de la recherche «, ANRW II.33.1, 304-386.

BINNS, J. W. ed. (1974), Latin Literature of the Fourth Century, London.

BURTON, P. J. (2000), Amicitia in Roman Social and International Relations (350-146 B.C.), diss., Univ. of Maryland.

BRUNT, P. (1988), The Fall of the Roman Republic and Related Essays, Oxford.

CALDELLI, M. L. (2001), «Amicus/-a nelle iscrizioni di Roma: I'apporto dell'epigrafia al chiarimento di un sentimento sociale», en Peachin, M. ed., 21-29.

CIMMA, M. R. (1976), Reges, Socii et Amici Populi Romani, Milano.

CITRONI MARCHETTI, S. (2000), Amicizia e Potere nelle Lettere di Cicerone e nelle Elegie Ovidiare dall Esilio, Firenze. 
Amistad y relaciones sociales en el Mundo Antiguo. Una aproximación al fenómeno...

CROOK, J. (1955), Consilium Principis, Cambridge.

DE BLOIS, L. (2001), "The political significance of friendship in the letters of Pliny the Younger», in Peachin, M. ed., 129-134

DE MARTINO, F. (1973), Storia della Costituzione Romana, II, Napoli.

DONLAN, W. J. (1985), «Pistos, philos, hetairos», en Figueira, T.J. y Nagy, G. eds., 223-244.

EVANS, K. G. (1997), «Friendship in Greek documentary papyri and inscriptions: a survey», en Fitzgeral, J.T. ed., 181-202.

FINLEY, M. I. (1961), El Mundo de Odiseo, México.

FIGUEIRA T. J. y NAGY, G. eds. (1985), Theognis of Megara: Poetry and the Polis, Baltimore.

FITZGERALD, J. T. ed. (1997), Greco-Roman Perspectives on Friendship, Atlanta.

FÜRST, A. (1996), Streit unter Freunden. Ideal und Realität in der Freundschaftslehre der Antike, Stuttgart.

HELLEGOUARC'H, J. (1972), Le Vocabulaire Latin des Relations et des Parties Politiques sous la République, Paris.

KONSTAN, D. (1997), Friendship in the Classical World, Cambridge.

MARCHALL, A. J. (1968), «Friends of the Roman people», AJP 89, 39-55.

MATTHEWS, F. J. (1974), «The Letter of Symmachus», en Binns, J.W. ed., 58-99.

MILLAR, F. (1977), The Emperor in the Roman World, London.

MONTES MIRALLES, M. Y. (2006), Ideología Aristocrática en los orígenes del arcaísmo griego: Estrategias de alteridad en la llíada, Oxford.

NARDUCCI, E. (2007), «Tito Pomponio Attico. Opinione su un amico. L'Antichità, il rinascimento, i Moderni», Bollettino di Studi Latini 37, 29-49.

PANCIERA, S. (2001), "Onorare l'amico nella sua casa. Amicitia e topografia a Roma e nel suo suburbio", en Peachin, M. ed., 11-19.

PEACHIN, M. ed. (2001), Aspects of Friendship in the Graeco-Roman World, Michigan.

PIZZOLATO, L. (1993), L'ldea di Amicizia nel Mondo Classico e Cristiano, Torino.

RAWSON, B. (1978), The Politics of Friendship. Pompey and Cicero, Sydney.

REALI, M. (1998), II Contributo dell'Epigrafia allo Studio dell'Amicitia. I/ Caso della Cisalpina, Firenze.

RODA, S. (1984), «Polifunzionalità della lettera comendaticia: teoria e prassi nell'epistolario simmachiano", en Roda S. ed., 225-255.

RODA, S. ed. (1994), La Parte Migliore del Genero Umano. Aristocrazie, Potere e Ideologia nell'Occidente Tardoantico, Torino.

ROULAND, N. (1979), Pouvoir Politique et Dépendance Personnelle dans l'Antiquité Romaine. Genèse et Rôle des Rapports de Clientèle, Bruxelles.

SALLER, R. P. (1982), Personal Patronage under the Early Empire, Cambridge.

- (1989), «Patronage and friendship in Early Imperial Rome: drawing the distinction», en Wallace-Hadrill, ed., 49-62.

SALZMAN, M. R. (2006), "Symmachus and the 'Barbarian generals'», Historia 55, 352-367.

SAVALLI-LESTRADE, I. (1998), Les Philoi Royaux dans l'Asie Hellénistique, Geneva.

SCHIEVENIN, R. (2000), «Amicizia perfecta e amicizia comune nel Laelius ciceroniano», Bolletino di Studi Latini 30, 447-466.

SERRANO DELGADO, J. M. (1987-1988), «La aportación de la epigrafía para el conocimiento de la amicitia-relación de dependencia en el alto imperio", Habis 18-19, 345-364.

- (1988), Estatus y Promoción Social de los Libertos en Hispania Romana, Sevilla.

SIVER, A. (1990), "Friendship in commercial society: eighteenth-century social theory and modern sociology", American Journal of Sociology 95, 1474-1504.

STERN-GILLET, S. (1995), Aristotle's Philosophy of Friendship, Albany.

SYME, R. (1937), The Roman Revolution, Oxford.

VERBOVEN, K. (2002), The Economy of Friends. Economic Aspects of Amicitia and Patronage in the Late Republic, Bruxelles.

WALLACE-HADRILL, A. ed. (1989), Patronage in Ancient Society, London. 
WHITE, C. (1992), Christian Friendship in the Fourth Century, Cambridge.

WHITLOCK BLUNDELL, M. (1989), Helping Friends and Harming Enemies. A Study in Sophocles and Greek Ethic, Cambridge.

WINTERLING, A. (2008), «Freundschft und Klientel im kaiserzeitlichen Rom», Historia 57, 218-316. 